\title{
Dynamics of plasma biomarkers in Down syndrome: the relative levels of $A \beta 42$ decrease with age, whereas NT1 tau and NfL increase
}

\section{David Mengel}

University of Tuebingen

Wen Liu

Brigham and Women's Hospital

Robert J. Glynn

Brigham and Women's Hospital

Dennis J. Selkoe

Brigham and Women's Hospital

Andre Strydom

King's College London

Florence Lai

Massachusetts General Hospital

H. Diana Rosas

Massachusetts General Hospital

Amy Torres

Massachusetts General Hospital

Vasiliki Patsiogiannis

Massachusetts General Hospital

Brian Skotko

Massachusetts General Hospital

Dominic Walsh ( $\nabla$ davidmengel@gmx.net)

Brigham and Women's Hospital and Harvard Medical School

\section{Research}

Keywords: Alzheimer's disease, amyloid, Down syndrome, dementia, neurofilament light, Simoa

Posted Date: January 29th, 2020

DOI: https://doi.org/10.21203/rs.2.18106/v2 
License: (c) (i) This work is licensed under a Creative Commons Attribution 4.0 International License. Read Full License

Version of Record: A version of this preprint was published at Alzheimer's Research and Therapy on March 19th, 2020. See the published version at https://doi.org/10.1186/s13195-020-00593-7. 


\section{Abstract}

Background Down syndrome (DS) is the most common genetic cause of Alzheimer's Disease (AD), but diagnosis of $A D$ in $D S$ is challenging due to the intellectual disability which accompanies DS. When disease-modifying agents for $A D$ are approved, reliable biomarkers will be required to identify when and how long people with DS should undergo treatment. Three cardinal neuropathological features characterize $A D$, and $A D$ in $D S$ - $A \beta$ amyloid plaques, tau neurofibrillary tangles, and neuronal loss. Here, we quantified plasma biomarkers of all 3 neuropathological features in a large cohort of people with DS aged from 3 months to 68 years.

Methods Using ultra-sensitive single molecule array (Simoa) assays, we measured 3 analytes ( $A \beta 42, N f L$, and tau) in plasmas of 100 individuals with DS and 100 age- and sex-matched controls. Tau was measured using an assay (NT1) which detects forms of tau containing at least residues 6-198, and the stability of the 3 analytes was established using plasma from ten healthy volunteers collected at 6 intervals over a five day period.

Results High $A \beta 42$ and NT1 tau, and low NfL, were observed in infants. Across all ages, $A \beta 42$ levels were higher in DS than controls. Levels of $A \beta 42$ decreased with age in both DS and controls, but this decrease was greater in DS than controls and became prominent in the third decade of life. NT1 tau fell in adolescents and young adults, but increased in older individuals with DS. NfL levels were low in infants, children, adolescents and young adults, but thereafter increased in DS compared to controls.

Conclusions High levels of $A \beta 42$ and tau in both young controls and DS suggest these proteins are produced by normal physiological processes, whereas, the changes seen in later life are consistent with emergence of pathological alterations. Our plasma biomarker results are in good agreement with prior neuropathology studies and indicate that the third and fourth decades (i.e. 20 to 40 years of age) of life are pivotal periods during which AD processes manifest in DS. Application of the assays used here to longitudinal studies of individuals with DS aged 20 to 50 years of age, should further validate the use of these biomarkers, and in time may allow identification and monitoring of people with DS best suited for treatment with emerging $A D$ therapies.

\section{Introduction}

Trisomy of chromosome 21, commonly known as Down syndrome (DS), is the most common genetic cause of lifelong intellectual disability, and occurs in over 7 million people worldwide [1]. DS is also the most common genetic cause of early onset Alzheimer's Disease (AD) [2], and in prospective studies the cumulative incidence for dementia is around 90\% [3-5]. Given that the AD-associated APP gene is on chromosome 21, and that rare DS individuals who lack a third copy of APP do not develop AD [6], overexpression of APP and accumulation of the amyloid $\beta$-protein $(A \beta)$ are considered the main drivers of AD in DS. 
Early clinical diagnosis of $A D$ in people with $D S$ is complex and challenging because patients with DS have a pre-existing intellectual disability, the extent of which varies from person to person. Reliable $A D$ specific biomarkers would greatly assist early diagnosis of AD in DS and could facilitate clinical care planning and timely treatment with emerging $A D$ therapies. Measurement of $A \beta$ and tau by their detection in CSF using immunoassays or visualization of deposited proteins using PET imaging, can reliably detect $A D$ in at-risk individuals [7-9]. However, PET imaging is expensive and its application outside of clinical research remains limited. Collection of CSF, although routine in certain countries, remains unpopular with patients, especially if required more than once. Lumbar puncture and PET scans are particularly challenging in a vulnerable population such as people with DS. In contrast, collection of blood would be significantly easier for individuals with DS, making blood-based biomarkers of AD in DS the preferred option.

Post-mortem studies of brains from individuals with DS reveal that in general, diffuse amyloid plaques appear in the late teens, and tau pathology emerges after age $35[10,11]$. Whether blood-based biomarkers of $A D$ evolve in a similar temporal pattern is not yet clear. Prior studies of putative $A D$ biomarkers examined restricted age groups [12-14], and earlier studies employed sub-optimal assays [15]. Most previous investigations on AD biomarkers in DS were limited to analysis of plasma $A \beta$. Now with the advent of reliable methods it is possible to assess whether tau and neurofilament light $(\mathrm{NfL})$ are also altered in DS. NfL, a scaffolding cytoskeleton protein, is elevated in plasma in many neurodegenerative conditions [16], and several NfL assays are available [17]. Measurement of tau in plasma has been less straightforward. This is because tau is molecularly heterogeneous [18-20] and is present in blood at only minute levels. We recently developed an ultra-sensitive immunoassay, which detects forms of tau captured by the mid-region antibody BT2 (to aa 194-198) and detected with the Nterminal antibody Tau12 (to aa 6-13). In several studies we have found that measurement of tau using this NT1 assay effectively discriminates AD from controls [19] and is elevated in patients with mild cognitive impairment who subsequently developed $A D$ [21]. Other assays which target distinct epitopes of tau have been reported but these have shown less consistent differences between AD and controls [2224].

Here, we measured AB42, NT1 tau, and NfL in plasma from individuals with DS and age-matched controls. Our study cohort covered a broad age-range, including participants as young as 3 months and as old as 68 years. The study had 2 primary objectives :1) to assess changes of plasma AB42, NT1 tau, and NfL in DS across age, and 2) to compare biomarkers measured in DS plasma versus age- and sexmatched controls. The usefulness of any biomarker is influenced by a myriad of factors, key among which are the stability of the analyte(s) over time and whether or not it is affected by diurnal factors and requires pre-sampling fasting. Prior to analyzing precious specimens from our unique DS-control matched cohort we examined the stability of analytes in the plasma of 10 healthy volunteers collected at 6 time points over a 5 day interval.

\section{Material And Methods}




\subsection{Participants}

\subsubsection{PRECISION study cohort}

Blood was collected from ten healthy volunteers twice a day at two-day intervals over a five-day period. Participant characteristics are presented in Supplemental Table 1 (Additional File 1). On each day, blood was collected in the morning between $7-10$ am and in the evening between $4-7 \mathrm{pm}$. The average time between blood draws on the same day was $8.4 \pm 1.0$ hours. Participants had fasted for at least 10 hours prior to morning blood draws (average fasting period: $11.2 \pm 1.0$ hours), but fasting was not requested for blood collection done in the evening. Blood was collected into $8 \mathrm{~mL}$ ethylenediaminetetraacetic acid (EDTA) treated tubes by peripheral venipuncture with a $21 \mathrm{G}$ butterfly needle. Plasma was processed as quickly as possible, and the average time between venipuncture to plasma isolation was 19 minutes. Specimens were centrifuged at 2,000 $\mathrm{g}$ for 10 minutes at $4{ }^{\circ} \mathrm{C}$, and the supernatant was carefully removed and aliquoted into $500 \mu \mathrm{L}$ lots in $1.5 \mathrm{~mL}$ Eppendorf protein lobind tubes and stored at $-80^{\circ} \mathrm{C}$. The study was conducted in accordance with local clinical research regulations and approved by the Partners Institutional Review Board (Walsh, BWH2017P000259), and all participants gave written informed consent.

\subsubsection{Down syndrome and control participants}

Plasma samples were taken from 100 people with Down syndrome. Samples were obtained from three centers. The bulk of the samples $(n=82)$ were collected prospectively at the Massachusetts General Hospital Down Syndrome Program. Additional samples came from the Center for Neuroimaging of Aging and Neurodegenerative Disease of the Massachusetts General Hospital $(n=13)$, and the LonDownS Consortium at King's College London $(n=5)$.

\section{Massachusetts General Hospital Down Syndrome Program}

Participants with Down Syndrome and/or their legal guardians were consented during outpatient visits at the Massachusetts General Hospital Down Syndrome Program. The protocol was approved by the Partners Human Research Committee. Blood was drawn from the antecubital vein with a $21 \mathrm{G}$ needle and collected into EDTA treated tubes. Plasma was isolated by centrifugation at $2000 \times g$ at $4^{\circ} \mathrm{C}$ for 10 minutes, and aliquots processed and stored as described for the PRECISION cohort.

Center for Neuroimaging of Aging and Neurodegenerative Disease of the Massachusetts General Hospital

Participants with DS were recruited from the MGH Down syndrome research database. Experimental procedures were explained and signed informed consent/assent was obtained prior to participation. The protocol was approved by the Institutional Review Board (2018P000898). Blood collection and processing was performed as described for the PRECISION study cohort. 
Participants with DS were also recruited as a part of the LonDownS Consortium's cohort study of Alzheimer's disease [25]. Ethical approval was obtained from the North West Wales Research Ethics Committee (13/WA/0194). Written informed consent was obtained if participants could consent for themselves, otherwise a consultee was asked to approve the individual's inclusion. Blood samples were collected in EDTA tubes and processed within 2 hours. Plasma was prepared by centrifuging samples for 10 minutes at $2,200 \times g$ at $4^{\circ} \mathrm{C}$; the supernatant was aliquoted and stored at $-80^{\circ} \mathrm{C}$.

\section{Boston Children's Hospital and Partners Biobank}

Specimens from age- and sex-matched control subjects were obtained from the Precision Link Biobank at Boston Children's Hospital (BCH) and the Biobank at Partners Healthcare in Boston (MA, USA). Controls had no history of diseases of the central nervous system. Specifically, there was no evidence of brain tumor, normal pressure hydrocephalus, stroke, severe brain trauma, brain surgery, epilepsy, encephalitis or dementia. Included participants were free of acute infectious disease.

Precision Link Biobank participants $(n=67)$ were enrolled throughout the hospital, across diverse clinical settings. In-person informed consent was obtained from all participants enrolling in the Biobank and provides permission to (1) access electronic health record data for research, (2) collect and use of residual specimens produced as by-products of routine care, and (3) share de-identified data and specimens outside of the institution. Participants provided a $4 \mathrm{~mL}$ blood sample for research use. Whole blood was collected in EDTA tubes, centrifuged at $2000 \times \mathrm{g}$ for 10 minutes at room temperature, with plasma removed and aliquoted into $0.5 \mathrm{~mL}$ microcentrifuge tubes. Aliquots were stored at $-80{ }^{\circ} \mathrm{C}$ in the Biobank Core Lab facility until requested. The Precision Link Biobank initiative is approved by the $\mathrm{BCH}$ Institutional Review Board (P00000159).

Additional samples ( $n=33)$ and health information were obtained from the Partners HealthCare Biobank, a biorepository of consented patient samples at Partners HealthCare. The Partners HealthCare Biobank is approved by the Institutional Review Board (2009P002312). Blood was collected, and plasma generated as described above.

\subsection{Single molecule array (Simoa) immunoassays}

Levels of AB42, NT1 tau, and NfL were quantified using Simoa assays. All assays were performed by the same operator and conducted on the same automated HD-1 analyzer (Quanterix, Billerica, MA).

Consumables and reagents other than certain antibodies were obtained from Quanterix. The NT1 tau assay was developed in-house [19], and commercial kits were used to measure A 342 and NfL.

NT1

This is a 3-step assay capable of detecting all forms of tau which contain residues 6 - 198 [19]. BT2 (194-198, Thermo, Waltham, MA, USA) was conjugated onto paramagnetic beads at $2 \mathrm{mg} / \mathrm{mL}$ and used for capture. Biotinylated Tau12 (6-13, Merck Millipore, Darmstadt, Germany) was used for detection. 
The optimal plasma dilution (1:4) to minimize matrix effects was determined previously [19]. Plasma samples were thawed on ice, centrifuged at $14,000 \times \mathrm{g}$ for 4 minutes, and the upper $90 \%$ of the supernatant transferred to Eppendorf protein lobind tubes and then diluted 1:4 with Tau 2.0 sample diluent reagent (Quanterix, Billerica, MA). Samples, standards and blanks were analyzed at least in duplicate.

The lower limit of quantification (LLOQ) defined as the lowest standard: (i) with a signal higher than the average signal for the blank plus 9 SDs, and (ii) allowed a percent recovery $\geq 100 \pm 20 \%$. In 4 runs over 4 days the LLoQ was $0.25 \mathrm{pg} / \mathrm{mL}$. The average \% coefficient of variation (\%CV) of all samples measured in the study was $13.8 \%$. Assay characteristics including selectivity, dilution linearity, and spike and recovery were reported previously [19].

$A \beta 42$ and $N f L$

The Simoa ${ }^{\circledR}$ A 442 Advantage (Quanterix, Billerica, MA, USA) and Simoa ${ }^{\text {TM }}$ NF-light ${ }^{\circledR}$ Advantage (Quanterix, Billerica, MA, USA) kits were used according to the manufacturer's instructions. Reagents from a single lot were used for analysis of all specimens from either the PRECISION study cohort or the Down syndrome study cohort. To determine the optimal dilution factor, plasma samples from 73 healthy donors were diluted 1:4 and 1:8 and analyzed for $A \beta 1-42$ and $N f L$, and the highest dilution factor that allowed reliable quantification of samples was used. Thereafter, specimens were diluted 1:8 for $A \beta 42$ and 1:4 for NfL. As with specimens for the NT1 assay, plasma was centrifuged at $14,000 \times g$ for 4 minutes, the upper $90 \%$ transferred to a new Eppendorf protein lobind tube and diluted with sample diluent provided in the kits. LLoQs for the AB1-42 and NfL assays were calculated as described for the NT1 assay, and were $0.41 \mathrm{pg} / \mathrm{mL}$ and $0.47 \mathrm{pg} / \mathrm{mL}$, respectively. The average \%CV for all samples measured in the study was $5.5 \%$ for the $A \beta 1-42$ and $7.5 \%$ for the $\mathrm{NfL}$ assay.

\subsection{Statistical analyses}

Statistical analyses were carried out using GraphPad Prism, version 8 (LaJolla, CA, USA) and Stata, version 15.1 (Stata Corp., College Station, TX, USA). The effect of daytime (morning vs. evening blood draws) and different weekdays (Day 1 vs. Day 3 vs. Day 5) on plasma biomarker levels was assessed using linear mixed models ("mixed"). Both variables were included as fixed affects, whereas subjects were used as random effects. To assess goodness-of-fit we included both variables in a stepwise process and compared Akaike Information criterion (AIC) of the respective models ("estat ic"). Tests on estimated coefficients were performed using the Wald test postestimation command ("test"). Differences of biomarker levels between DS and controls for specified age-ranges were assessed using a paired $t$-test (for normally distributed data) or a Wilcoxon signed-rank test (for non-normally distributed data). $p$ values were adjusted for multiple testing by Bonferroni correction. Additionally, piecewise linear regression using hockey-stick regression was applied to model the effect of age on plasma biomarker levels in DS and controls (and differences between both groups) [26]. The slopes of both regression lines and the change-point where both regression lines meet were estimated using the "nl hockey" function. From specimens of DS and age-matched control participants, 10 out of 600 measurements $(1.7 \%, 7$ for 
the NT1 tau and 3 for the NfL assay, see Additional File 1: Supplemental Table 2 for raw data) were below the LLoQ of the respective assay (determined as described above), and for statistical analysis these samples were assigned values equal to the LLoQ of the assay. Statistical analysis was repeated with the actual readings of these 10 measurements, but produced similar results. The significance threshold was set to a two-sided $p \leq 0.05$.

\section{Results}

\section{Plasma A $342, \mathrm{NT1}$ and NfL levels are stable over a 5 day period and are not influenced by the time of day when blood is collected}

The usefulness of any biomarker is, amongst other factors, influenced by whether it: (i) remains constant over a reasonable period of time, (ii) is altered by diurnal factors, and (iii) requires pre-sampling fasting. Here we sought to examine biomarkers of amyloid, tau and neurodegeneration [27] in plasma of individuals with DS across the first seven decades of life. Before analyzing precious clinical samples, we conducted experiments to test the stability of analytes to be measured, namely Aß42, NT1 tau, and NfL. Blood was collected from 10 healthy volunteers on day 1,3 and 5. On each collection day, blood was obtained at two time-points: (i) morning (after an over-night fasting), and (ii) evening. This design was employed to account for routine clinical practice in an out-patient setting in which patients are either asked to donate blood in the morning after over-night fasting, or to attend clinic later in the day without any dietary restrictions. Figure 1 shows the measured concentrations of A $A 42, N T 1$ tau, and NfL in the morning (red circles) and in the evening (blue squares). Spaghetti-blots of individual measurements from all 10 healthy volunteers are presented in Supplemental Figures $1-3$ (Additional File 2). Measurements for all three analytes in specimens collected in the morning and in the evening on three different days in one week were highly similar (Figure 1). There was no statistically significant effect of time of day (morning vs. evening), and analyte levels were stable across the 5 day sampling period (Day 1 vs. Day 3 vs Day 5 ). The $\% C V$ s of measurements obtained on the morning of day 1 , day 3 , and day 5 in the same individual for A $\mathrm{A} 42$, NT1 tau and NfL were 3.8\% (range $0.9-6.2 \%$ ), 9.3\% (range $1.3-20.3 \%$ ) and $7.3 \%$ (range $0.4-15.0 \%$ ), respectively. Comparable \%CVs were observed for measurements in the evening (Table 1). Collectively, these results demonstrate that the forms of $A \beta$, tau, and $\mathrm{NfL}$ in plasma measured by the A $342, N T 1$ tau, and NfL assay are stable, and do not appear to be influenced by diurnal and dietary factors.

\section{$A \beta 1-42, N T 1$, and NfL plasma concentrations depend on age in Down syndrome individuals compared to controls}

To study age-dependent changes of plasma AD biomarkers in DS, samples were obtained from 100 people with DS aged 3 months to 68 years. One hundred age-and sex-matched participants free of neurological disease served as controls (see Table 2 for participant characteristics). 
Individual results for all 3 analytes are presented in Supplemental Table 2 (Additional File 1). Concentrations of $A \beta 42$ were higher in individuals with DS than controls across the entire age-range (Figure $2 A$ and $D$ and Table 3 ). In the youngest age group ( $0-10$ years), mean $A \beta 42$ concentrations were approximately 1.6-fold higher in DS individuals compared to controls ( $45.3 \mathrm{vs}$. $28.1 \mathrm{pg} / \mathrm{mL}, \mathrm{p}<0.001$ ), but the differences between plasma A $1-42$ levels in individuals with DS and controls declined with increasing age (Figure $3 A$ and $D$ ). At approximately 30 years of age $A \beta 42$ levels reached a steady level of $\sim 22 \mathrm{pg} / \mathrm{mL}$ for individuals with $\mathrm{DS}$, and $\sim 15 \mathrm{pg} / \mathrm{mL}$ for controls. In the oldest age group (>50 years), mean A 42 concentrations were 1.4-fold higher in individuals with DS compared to controls (22.7 $\mathrm{pg} / \mathrm{mL}$ vs. $16.6 \mathrm{pg} / \mathrm{mL}, \mathrm{p}=0.07)$.

For NT1 tau, levels were highest in children and fell with age in both the DS and control groups (Figure 2 $B$ and $E$ and Table 3). In general, the levels of NT1 tau in DS and controls broadly overlapped, but tended

to diverge after the age of 30 (Figure $3 \mathrm{~B}$ and $\mathrm{E}$ and Table 3). Specifically, in individuals with DS 50 years and older, NT1 levels were significantly increased compared to controls $(7.0 \mathrm{vs.} 4.0 \mathrm{pg} / \mathrm{mL}, \mathrm{p}=0.042)$ (Figure $3 \mathrm{E}$, Table 3).

For the first three decades of life, plasma NfL levels were relatively constant and were similar in DS and controls (Figure $2 \mathrm{C}$ and $\mathrm{F}$ and Figure $3 \mathrm{C}$ and F, Table 3). In subjects over 30 years of age, NfL levels increased in both controls and DS, but the increase was considerably greater for people with DS.

Specifically, NfL levels were significantly increased in individuals with DS compared to controls at ages 40 and older ( $41-50$ years: 15.9 vs. $8.3 \mathrm{pg} / \mathrm{mL}, \mathrm{p}=0.001 ;>50$ years: 31.0 vs. $11.7, \mathrm{p}=0.023$ ) (Figure $2 \mathrm{~F}$, Table 3).

We also applied more sophisticated statistical models in an attempt to better understand differences in the age-dependent changes in DS versus controls. Specifically, we used piece-wise linear regression to determine time-points of slope-changes in DS and controls (Additional File 1: Supplemental Table 3 and Additional File 2: Supplemental Figure 4). However, some estimates for time-points of slope change were associated with relatively wide confidence intervals making it problematic to assign reliable change points.

\section{Discussion}

The age-dependent accrual of amyloid plaques and neurofibrillary tangles occurs in all individuals with complete trisomy of chromosome $21[10,11]$, and after age 50 , cognitive deterioration consistent with AD dementia is common in people with Down syndrome [28]. Because individuals with DS have varying degrees of intellectual disability, it is often difficult to use cognitive measures in a systematic and reliable way to determine the clinical onset of $A D$. Thus, the discovery of reliable $A D$ biomarkers is critical for the future clinical management and treatment of $A D$ in DS. With the potential of disease modifying treatments in sight $[29,30]$ it will become increasingly important to identify DS individuals developing AD who might benefit from emerging interventions. 
Here, we sought to assess changes in biomarkers related to 3 primary features of AD: amyloid, tau, and neurodegeneration [27]. In the absence of large longitudinal cohorts, we gathered specimens from 100 DS individuals aged from 3 months to 68 years and compared their values with those of age- and sexmatched controls. As expected for individuals with 3 copies of the APP gene (and consistent with prior studies [15, 31-33]) we observed higher plasma $A \beta 42$ levels in individuals with DS compared to controls. In the first decade of life, a time when there is little amyloid deposition [34, 35], plasma A 342 levels were on average a little higher ( 1.6 fold) in DS than the expected 1.5-fold elevation due to gene dosage. Why this should be is unclear, but it is worth noting that several AD risk factors are encoded on chromosome 21 [2] and these might contribute to either enhanced amyloidogenic processing of APP [36, 37] or reduced degradation of $A \beta$ [38]. Also, neuronal $A \beta$ production is activity dependent [39] and in DS there is evidence of aberrant hyperactivity during development and early life [40] that could contribute to higher $A \beta$ levels.

$A \beta 42$ levels tended to fall with age in both individuals with DS and controls, but the relative decrease was greater in DS. There was a strong tendency for the DS/control AB42 ratio to be lower in the oldest group ( $>50$ years) compared to youngest group ( 0 - 10 years), however, this did not reach statistical significance (1.6 vs. $1.4, p=0.08$ ). Nonetheless, the trend is consistent with the notion that $A \beta 42$ is prone to aggregate and becomes trapped in accumulating plaques and is in line with multiple AD studies linking falling CSF and plasma A 42 with increased cerebral amyloid deposition [41, 42]. However, prior studies examining the association of plasma $A \beta 42$ with age in DS have yielded conflicting results with reports of increased [43], decreased [44], and unchanged $A \beta 42$ levels [12,32, 45]. But previous studies were not designed to look at the effect of age across a broad age-span. We found that plasma $A \beta 42$ levels fell sharply in the first 3 decades of life in individuals with DS, but were relatively stable in the age-range from 31 to 68 years. Our results gathered using specimens from a broad age range of individuals ( 3 months to 68 years) provide the perspective to better understand what had formerly appeared discordant results, that is decreasing AB42 levels in younger DS individuals [44] but relatively stable levels in older DS individuals $[12,32,45]$.

Human plasma is a complex matrix, components of which can interfere with immunoassays. One means of overcoming matrix interference is to dilute samples so as to reduce interfering plasma components to a level below which they no longer interfere. This requires that the assays employed are sufficiently sensitive to allow dilutions necessary to preclude matrix interference. Here, we employed ultra-sensitive assays and evaluated the maximum dilution that allowed consistent detection of analytes across a large number of human samples. For $A \beta 42$, only a few studies $[25,33,46,47]$ have used such ultra-sensitive techniques.

In contrast to $A \beta$, only a handful of studies have attempted to measure tau in plasma of DS individuals. Extracellular tau is molecularly complex [18-20] and different assays detect distinct populations of tau alloforms, complicating comparisons of results obtained using different assays. Here, we utilized our inhouse NT1 tau assay which we have previously shown to be capable of detecting forms of tau that are significantly elevated in plasma of patients with AD-MCl and mild AD [19]. Like Aß42, NT1 detected tau 
was highest at early age (0-10 years). NT1 levels fell between 11 and 30 years but thereafter increased. This pattern is a mirror image of the $A \beta 42$ results, with both exhibiting pivotal changes between 20 and 40 years.

$\mathrm{NfL}$, a now widely validated marker of neurodegeneration [16], was relatively low in early life, but in both controls and DS, NfL increased steadily after age 30. Importantly, NfL levels in plasma of individuals with DS started to diverge from control levels in the 31-40 years age group and were most different in the two oldest age groups.

Collectively, our findings demonstrate that plasma measures of amyloid, tau and neurodegeneration change with age and that the relative differences in these markers are greatest in the 31-40, 41-50 and over 50 years age groups. In our study cohort, elevated concentrations of NfL and NT1 measured tau in older individuals with DS are consistent with: (i) recent cross-sectional studies that found increased plasma NfL and tau in prodromal and AD dementia in people with DS [33,48], and (ii) an increasing prevalence of $A D$ in older individuals with $D S[4,5]$. Notwithstanding the identification of these important trends, our results indicate that it will be impractical to use a single time point measurement of these biomarkers to diagnose AD in DS. Rather, our data support longitudinal assessment of these markers to further evaluate their potential to predict onset of disease.

A particular strength of this study is the use of a relatively large number of DS individuals $(n=100)$ and age- and sex-matched controls ( $n=100)$ with a broad age-range ( 3 months to 68 years). Another strength is the use of analytically validated methods and testing conditions. The major weaknesses of our study include the fact that the study is cross-sectional and not longitudinal, the use of controls from a biobank, and that we did not include cognitive assessments. Future studies should collect clinical information, such as cognitive measures, APOE status and concomitant medication, and it may be useful to measure other alloforms of $A \beta$ so as to calculate ratios of different $A \beta$ species (e.g. $A \beta 42 / 40$ ).

\section{Conclusions}

Viewed together, the trajectories of all three biomarkers point towards important changes after the third decade of life in people with DS. During that period, AB42 in DS falls considerably and NT1 and NfL levels rise steadily. In order to identify individuals with DS who would most benefit from secondary prevention treatments, future prospective longitudinal studies should focus on the critical age span between 30 and 50 years measuring both biomarker changes and cognition. Our successful use of sensitive and dynamic plasma analytes reflecting the cardinal features of $A D$ is particularly salient for people with DS, given the ethical challenges of trying to use CSF or brain imaging to monitor their temporal progression into AD.

\section{List Of Abbreviations}

Aß42: Amyloid- $\beta$ protein ending at amino acid 42

Page $11 / 23$ 
AD: Alzheimer's Disease

APOE: Apolipoprotein E

APP: Amyloid precursor protein

CSF: Cerebrospinal fluid

DS: Down syndrome

EDTA: Ethylenediaminetetraacetic acid

LLoQ: Lower limit of quantitation

NT1 tau: N-terminal tau 1 assay (detects forms of tau containing at least residues 6-198)

NfL: Neurofilament light

PET: Positron emission tomography

Simoa: Single molecule array

\%CV: Percent coefficient of variation

\section{Declarations}

\section{Ethics approval and consent to participate}

Signed informed consent/assent was obtained prior to participation from all individuals. Protocols were approved by the Internal Review Board of all participating research sites (Partners Intitutional Review Board, BCH Institutional Review Board, North West Wales Research Ethics Committee).

\section{Consent for publication}

Not applicable.

\section{Availability of data and materials}

Raw data of plasma measurements in DS and controls are reported in Supplemental Table 2 (Additional File 1). All other data supporting the conclusions of this manuscript will be made available by the corresponding authors, without undue reservation, to any qualified researcher. All materials used in the publication are commercially available. 


\section{Competing interests}

DMW is an employee of Biogen Inc. AS has consulted for/ or been an investigator on studies sponsored by the following companies: Hoffmann-la Roche Itd, ONO Pharma, Aelis Farma, AC-Immune, and ProMIS Neurosciences. DJS is a director and consultant to Prothena Biosciences. BS occasionally consults on the topic of Down syndrome through Gerson Lehrman Group. He receives remuneration from Down syndrome non-profit organizations for speaking engagements and associated travel expenses. BS receives annual royalties from Woodbine House, Inc., for the publication of his book, Fasten Your Seatbelt: A Crash Course on Down Syndrome for Brothers and Sisters. Within the past two years, he has received research funding from F. Hoffmann-La Roche, Inc. and LuMind Research Down Syndrome Foundation to conduct clinical trials for people with Down syndrome. BS is occasionally asked to serve as an expert witness for legal cases where Down syndrome is discussed. BS serves in a non-paid capacity on the Honorary Board of Directors for the Massachusetts Down Syndrome Congress and the Professional Advisory Committee for the National Center for Prenatal and Postnatal Down Syndrome Resources. BS has a sister with Down syndrome. None of the other authors have biomedical financial interests or potential conflicts of interest.

\section{Funding}

DMW is an Alzheimer's Association Zenith fellow and this work was made possible by support to DMW from the Alzheimer's Association, the Alzheimer's Drug Discovery Foundation, and the Foundation for Neurologic Diseases; and to AS from the Wellcome Trust Strategic Award (grant number: 098330/Z/12/Z) conferred upon The London Down Syndrome (LonDownS) Consortium. DM is supported by a Research Fellowship from the German Research Foundation (DFG ME 4858/1-1).

\section{Author contributions}

DMW conceived the project, designed and supervised the research. DMW and DM wrote the manuscript. BS assisted in the design of the experiments. DM wrote study protocols, collected samples, carried out the experiments, analyzed data and prepared the Figures. WL assisted with the processing and analysis of samples. AS supplied archived samples. FL, HDR, DJS, and BS, AT, and VP provided samples. RJG assisted with statistical analysis. All the authors critically assessed and contributed to writing the manuscript.

\section{Acknowledgments}

Page 13/23 
We are grateful to blood donors and their families and clinician colleagues for referring patients and collecting blood. We thank Partners HealthCare Biobank for providing samples, and health information data. We also acknowledge material and data support from the Precision Link Biobank for Health Discovery at Boston Children's Hospital.

\section{Tables}

Table 1: Biological Variation of Analytes

\begin{tabular}{|c|c|c|c|}
\hline Assay & Daytime & $\begin{array}{c}\text { Mean } \pm \text { SD (range) level for all 10 } \\
\text { donors }(\mathrm{pg} / \mathrm{mL})\end{array}$ & $\begin{array}{c}\text { Mean } \pm \text { SD (range) intra-subject \%CV for } \\
\text { all 10 donors }\end{array}$ \\
\hline A 342 & Morning & $20.07 \pm 2.81(16.1-26.2)$ & $3.8 \pm 1.9 \%(0.9-6.2 \%)$ \\
& Evening & $20.47 \pm 2.89(16.3-25.1)$ & $5.1 \pm 3.4 \%(1.0-12.2 \%)$ \\
\hline NT1 & Morning & $2.31 \pm 0.49(1.4-3.4)$ & $9.3 \pm 5.3 \%(1.3-20.3 \%)$ \\
tau & Evening & $2.26 \pm 0.51(1.5-3.5)$ & $8.7 \pm 7.3 \%(1.1-26.5 \%)$ \\
\hline NfL & Morning & $7.20 \pm 3.30(2.4-13.4)$ & $7.3 \pm 4.7 \%(0.4-15.0 \%)$ \\
& Evening & $7.09 \pm 3.03(2.5-13.7)$ & $8.9 \pm 4.3 \%(3.3-17.1 \%)$ \\
\hline
\end{tabular}

Abbreviations: SD, standard deviation; \%CV, percentage coefficient of variation

Table 2: Demographics of DS and Controls 


\begin{tabular}{|c|c|c|c|c|}
\hline & & & Down syndrome & Control \\
\hline & $\begin{array}{l}\text { Age groups (in } \\
\text { years) }\end{array}$ & $\mathrm{N}$ & $\begin{array}{c}\text { Age in years } \\
\text { SD }\end{array}$ & $\begin{array}{ll}\text { Age in years } & \text { mean } \pm \\
S D & \end{array}$ \\
\hline \multirow{7}{*}{$\begin{array}{l}\text { Mean Age } \\
\text { (year) }\end{array}$} & $0-10$ & 30 & $4.9 \pm 2.9$ & $5.0 \pm 2.9$ \\
\hline & $11-20$ & 23 & $16.2 \pm 3.1$ & $16.3 \pm 3.2$ \\
\hline & $21-30$ & 15 & $24.1 \pm 2.8$ & $24.0 \pm 3.2$ \\
\hline & $31-40$ & 10 & $35.7 \pm 2.9$ & $35.5 \pm 3.0$ \\
\hline & $41-50$ & 13 & $45.4 \pm 3.6$ & $45.2 \pm 3.3$ \\
\hline & $>50$ & 9 & $57.1 \pm 5.6$ & $57.1 \pm 5.6$ \\
\hline & $\begin{array}{l}\text { Age group (in } \\
\text { years) }\end{array}$ & $\mathrm{N}$ & $\begin{array}{l}\text { Number of females (\% of } \\
\text { total) }\end{array}$ & $\begin{array}{l}\text { Number of females (\% of } \\
\text { total) }\end{array}$ \\
\hline \multirow[t]{6}{*}{ Sex (female) } & $0-10$ & 30 & $15(50.0)$ & $15(50.0)$ \\
\hline & $11-20$ & 23 & $10(43.5)$ & $10(43.5)$ \\
\hline & $21-30$ & 15 & $4(26.7)$ & $4(26.7)$ \\
\hline & $31-40$ & 10 & $4(40.0)$ & $4(40.0)$ \\
\hline & $41-50$ & 13 & $4(30.8)$ & $4(30.8)$ \\
\hline & $>50$ & 9 & $1(11.1)$ & $1(11.1)$ \\
\hline
\end{tabular}

Abbreviation: SD, standard deviation

Table 3: Biomarkers stratified by 10 year age group 


\begin{tabular}{|c|c|c|c|c|}
\hline Analyte & Age group (in years) & Down syndrome & Control & $p$-value* \\
\hline \multicolumn{5}{|l|}{$\mathrm{A} \beta 42(\mathrm{pg} / \mathrm{mL})$} \\
\hline & $0-10$ & $45.3 \pm 2.1$ & $28.1 \pm 1.6$ & $<0.001$ \\
\hline & $11-20$ & $31.8 \pm 2.0$ & $16.2 \pm 1.2$ & $<0.001$ \\
\hline & $21-30$ & $28.1 \pm 2.3$ & $15.7 \pm 0.8$ & $<0.001$ \\
\hline & $31-40$ & $21.4 \pm 1.3$ & $15.0 \pm 1.5$ & 0.014 \\
\hline & $41-50$ & $22.7 \pm 0.7$ & $14.7 \pm 0.6$ & $<0.001$ \\
\hline & $>50$ & $22.7 \pm 1.8$ & $16.6 \pm 0.8$ & n.s. \\
\hline \multicolumn{5}{|l|}{ NT1 tau $(\mathrm{pg} / \mathrm{mL})$} \\
\hline & $0-10$ & $8.3 \pm 0.5$ & $7.9 \pm 0.8$ & n.s. \\
\hline & $11-20$ & $4.3 \pm 0.5$ & $5.6 \pm 0.5$ & n.s. \\
\hline & $21-30$ & $4.3 \pm 0.4$ & $4.6 \pm 0.4$ & n.s. \\
\hline & $31-40$ & $4.2 \pm 0.6$ & $3.2 \pm 0.6$ & n.s. \\
\hline & $41-50$ & $4.6 \pm 0.4$ & $3.2 \pm 0.3$ & n.s. \\
\hline & $>50$ & $7.0 \pm 0.8$ & $4.1 \pm 0.2$ & 0.042 \\
\hline \multicolumn{5}{|l|}{$\mathrm{NfL}(\mathrm{pg} / \mathrm{mL})$} \\
\hline & $0-10$ & $7.5 \pm 0.5$ & $6.3 \pm 0.6$ & n.s. \\
\hline & $11-20$ & $5.1 \pm 0.4$ & $3.6 \pm 0.3$ & 0.010 \\
\hline & $21-30$ & $6.7 \pm 0.4$ & $5.4 \pm 0.9$ & n.s. \\
\hline & $31-40$ & $10.2 \pm 1.6$ & $6.8 \pm 1.3$ & n.s. \\
\hline & $41-50$ & $15.9 \pm 1.0$ & $8.3 \pm 1.0$ & 0.001 \\
\hline & $>50$ & $31.0 \pm 6.0$ & $11.7 \pm 1.1$ & 0.023 \\
\hline
\end{tabular}

mean concentrations \pm SEM; ${ }^{*} p$-value adjusted for multiple comparisons in age-strata by Bonferroni correction

\section{References}

1. Ballard C, Mobley W, Hardy J, Williams G, Corbett A: Dementia in Down's syndrome. Lancet Neurol 2016, 15:622-636. 
2. Wiseman FK, Al-Janabi T, Hardy J, Karmiloff-Smith A, Nizetic D, Tybulewicz VL, Fisher EM, Strydom A: A genetic cause of Alzheimer disease: mechanistic insights from Down syndrome. Nat Rev Neurosci 2015, 16:564-574.

3. McCarron M, McCallion P, Reilly E, Dunne P, Carroll R, Mulryan N: A prospective 20-year longitudinal follow-up of dementia in persons with Down syndrome. J Intellect Disabil Res 2017, 61:843-852.

4. Lai F, Williams RS: A prospective study of Alzheimer disease in Down syndrome. Arch Neurol 1989, 46:849-853.

5. Evenhuis HM: The natural history of dementia in Down's syndrome. Arch Neurol 1990, 47:263-267.

6. Doran E, Keator D, Head E, Phelan MJ, Kim R, Totoiu M, Barrio JR, Small GW, Potkin SG, Lott IT: Down Syndrome, Partial Trisomy 21, and Absence of Alzheimer's Disease: The Role of APP. J Alzheimers Dis 2017, 56:459-470.

7. Blennow K: A Review of Fluid Biomarkers for Alzheimer's Disease: Moving from CSF to Blood. Neurol Ther 2017, 6:15-24.

8. Handels RLH, Vos SJB, Kramberger MG, Jelic V, Blennow K, van Buchem M, van der Flier W, FreundLevi Y, Hampel H, Olde Rikkert $M$, et al: Predicting progression to dementia in persons with mild cognitive impairment using cerebrospinal fluid markers. Alzheimers Dement 2017, 13:903-912.

9. Villemagne VL, Dore V, Burnham SC, Masters CL, Rowe CC: Imaging tau and amyloid-beta proteinopathies in Alzheimer disease and other conditions. Nat Rev Neurol 2018, 14:225-236.

10. Davidson YS, Robinson A, Prasher VP, Mann DMA: The age of onset and evolution of Braak tangle stage and Thal amyloid pathology of Alzheimer's disease in individuals with Down syndrome. Acta Neuropathol Commun 2018, 6:56.

11. Mann DM: Cerebral amyloidosis, ageing and Alzheimer's disease; a contribution from studies on Down's syndrome. Neurobiol Aging 1989, 10:397-399; discussion 412-394.

12. Jones EL, Hanney M, Francis PT, Ballard CG: Amyloid beta concentrations in older people with Down syndrome and dementia. Neurosci Lett 2009, 451:162-164.

13. Abdel-Meguid IS, Abdel-Salam E, Latif DMA, Korra S, Ismaiel A: Markers of neural degeneration and regeneration in Down syndrome patients. The Egyptian Journal of Medical Human Genetics 2013, 14:49-53.

14. Conti E, Galimberti G, Piazza F, Raggi ME, Ferrarese C: Increased soluble APPalpha, Abeta 1-42, and anti-Abeta 1-42 antibodies in plasma from down syndrome patients. Alzheimer Dis Assoc Disord 2010, 24:96-100.

15. Alhajraf F, Ness D, Hye A, Strydom A: Plasma amyloid and tau as dementia biomarkers in Down syndrome: Systematic review and meta-analyses. Dev Neurobiol 2019.

16. Bridel C, van Wieringen WN, Zetterberg H, Tijms BM, Teunissen CE, Alvarez-Cermeno JC, Andreasson U, Axelsson M, Backstrom DC, Bartos A, et al: Diagnostic Value of Cerebrospinal Fluid Neurofilament Light Protein in Neurology: A Systematic Review and Meta-analysis. JAMA Neurol 2019. 
17. Kuhle J, Barro C, Andreasson U, Derfuss T, Lindberg R, Sandelius A, Liman V, Norgren N, Blennow K, Zetterberg $\mathrm{H}$ : Comparison of three analytical platforms for quantification of the neurofilament light chain in blood samples: ELISA, electrochemiluminescence immunoassay and Simoa. Clin Chem Lab Med 2016, 54:1655-1661.

18. Kanmert D, Cantlon A, Muratore CR, Jin M, O'Malley TT, Lee G, Young-Pearse TL, Selkoe DJ, Walsh DM: C-Terminally Truncated Forms of Tau, But Not Full-Length Tau or Its C-Terminal Fragments, Are Released from Neurons Independently of Cell Death. J Neurosci 2015, 35:10851-10865.

19. Chen Z, Mengel D, Keshavan A, Rissman RA, Billinton A, Perkinton M, Percival-Alwyn J, Schultz A, Properzi $\mathrm{M}$, Johnson $\mathrm{K}$, et al: Learnings about the complexity of extracellular tau aid development of a blood-based screen for Alzheimer's disease. Alzheimers Dement 2019, 15:487-496.

20. Cicognola C, Brinkmalm G, Wahlgren J, Portelius E, Gobom J, Cullen NC, Hansson O, Parnetti L, Constantinescu $\mathrm{R}$, Wildsmith $\mathrm{K}$, et al: Novel tau fragments in cerebrospinal fluid: relation to tangle pathology and cognitive decline in Alzheimer's disease. Acta Neuropathol 2019, 137:279-296.

21. Mengel D, Janelidze S, Liu W, Galasko D, Rissman RA, Selkoe DJ, Bennett DA, Hansson O, Walsh DM: Plasma NfL and NT1 predict conversion of $\mathrm{MCl} / \mathrm{SCl}$ to $\mathrm{AD}$ dementia. unpublished results.

22. Deters KD, Risacher SL, Kim S, Nho K, West JD, Blennow K, Zetterberg H, Shaw LM, Trojanowski JQ, Weiner MW, Saykin AJ: Plasma Tau Association with Brain Atrophy in Mild Cognitive Impairment and Alzheimer's Disease. J Alzheimers Dis 2017, 58:1245-1254.

23. Mattsson N, Zetterberg H, Janelidze S, Insel PS, Andreasson U, Stomrud E, Palmqvist S, Baker D, Tan Hehir CA, Jeromin A, et al: Plasma tau in Alzheimer disease. Neurology 2016, 87:1827-1835.

24. Olsson B, Lautner R, Andreasson U, Ohrfelt A, Portelius E, Bjerke M, Holtta M, Rosen C, Olsson C, Strobel G, et al: CSF and blood biomarkers for the diagnosis of Alzheimer's disease: a systematic review and meta-analysis. Lancet Neurol 2016, 15:673-684.

25. Startin CM, Ashton NJ, Hamburg S, Hithersay R, Wiseman FK, Mok KY, Hardy J, Lleo A, Lovestone S, Parnetti L, et al: Plasma biomarkers for amyloid, tau, and cytokines in Down syndrome and sporadic Alzheimer's disease. Alzheimers Res Ther 2019, 11:26.

26. nlhockey [personalpages.manchester.ac.uk/staff/mark.lunt/stata.html]

27. Jack CR, Jr., Bennett DA, Blennow K, Carrillo MC, Dunn B, Haeberlein SB, Holtzman DM, Jagust W, Jessen F, Karlawish J, et al: NIA-AA Research Framework: Toward a biological definition of Alzheimer's disease. Alzheimers Dement 2018, 14:535-562.

28. Sinai A, Mokrysz C, Bernal J, Bohnen I, Bonell S, Courtenay K, Dodd K, Gazizova D, Hassiotis A, Hillier $\mathrm{R}$, et al: Predictors of Age of Diagnosis and Survival of Alzheimer's Disease in Down Syndrome. $J$ Alzheimers Dis 2018, 61:717-728.

29. 'Reports of My Death Are Greatly Exaggerated.' Signed, Aducanumab [https://www.alzforum.org/news/research-news/reports-my-death-are-greatly-exaggerated-signedaducanumab]

30. Topline Results: 18 Months of BAN2401 Might Work [https://www.alzforum.org/news/researchnews/topline-results-18-months-ban2401-might-work] 
31. Petersen ME, O'Bryant SE: Blood-based biomarkers for Down syndrome and Alzheimer's disease: A systematic review. Dev Neurobio/ 2019.

32. Schupf N, Patel B, Silverman W, Zigman WB, Zhong N, Tycko B, Mehta PD, Mayeux R: Elevated plasma amyloid beta-peptide 1-42 and onset of dementia in adults with Down syndrome. Neurosci Lett 2001, 301:199-203.

33. Fortea J, Carmona-Iragui M, Benejam B, Fernandez S, Videla L, Barroeta I, Alcolea D, Pegueroles J, Munoz L, Belbin O, et al: Plasma and CSF biomarkers for the diagnosis of Alzheimer's disease in adults with Down syndrome: a cross-sectional study. Lancet Neurol 2018, 17:860-869.

34. Wisniewski KE, Wisniewski HM, Wen GY: Occurrence of neuropathological changes and dementia of Alzheimer's disease in Down's syndrome. Ann Neuro/ 1985, 17:278-282.

35. Lemere CA, Blusztajn JK, Yamaguchi H, Wisniewski T, Saido TC, Selkoe DJ: Sequence of deposition of heterogeneous amyloid beta-peptides and APO E in Down syndrome: implications for initial events in amyloid plaque formation. Neurobiol Dis 1996, 3:16-32.

36. Wegiel J, Gong CX, Hwang YW: The role of DYRK1A in neurodegenerative diseases. FEBS J 2011, 278:236-245.

37. Branca C, Shaw DM, Belfiore R, Gokhale V, Shaw AY, Foley C, Smith B, Hulme C, Dunckley T, Meechoovet B, et al: Dyrk1 inhibition improves Alzheimer's disease-like pathology. Aging Cel/ 2017, 16:1146-1154.

38. Yang DS, Stavrides P, Mohan PS, Kaushik S, Kumar A, Ohno M, Schmidt SD, Wesson D, Bandyopadhyay $U$, Jiang $Y$, et al: Reversal of autophagy dysfunction in the TgCRND8 mouse model of Alzheimer's disease ameliorates amyloid pathologies and memory deficits. Brain 2011, 134:258277.

39. Cirrito JR, Yamada KA, Finn MB, Sloviter RS, Bales KR, May PC, Schoepp DD, Paul SM, Mennerick S, Holtzman DM: Synaptic activity regulates interstitial fluid amyloid-beta levels in vivo. Neuron 2005, 48:913-922.

40. Arya R, Kabra M, Gulati S: Epilepsy in children with Down syndrome. Epileptic Disord 2011, 13:1-7.

41. Nakamura A, Kaneko N, Villemagne VL, Kato T, Doecke J, Dore V, Fowler C, Li QX, Martins R, Rowe C, et al: High performance plasma amyloid-beta biomarkers for Alzheimer's disease. Nature 2018, 554:249-254.

42. Palmqvist S, Janelidze S, Stomrud E, Zetterberg H, Karl J, Zink K, Bittner T, Mattsson N, Eichenlaub U, Blennow K, Hansson O: Performance of Fully Automated Plasma Assays as Screening Tests for Alzheimer Disease-Related beta-Amyloid Status. JAMA Neurol 2019.

43. Schupf N, Patel B, Pang D, Zigman WB, Silverman W, Mehta PD, Mayeux R: Elevated plasma betaamyloid peptide Abeta(42) levels, incident dementia, and mortality in Down syndrome. Arch Neurol 2007, 64:1007-1013.

44. Mehta PD, Mehta SP, Fedor B, Patrick BA, Emmerling M, Dalton AJ: Plasma amyloid beta protein 1-42 levels are increased in old Down Syndrome but not in young Down Syndrome. Neurosci Lett 2003, 342:155-158. 
45. Matsuoka Y, Andrews HF, Becker AG, Gray AJ, Mehta PD, Sano MC, Dalton AJ, Aisen PS: The relationship of plasma Abeta levels to dementia in aging individuals with Down syndrome. Alzheimer Dis Assoc Disord 2009, 23:315-318.

46. Lee NC, Yang SY, Chieh JJ, Huang PT, Chang LM, Chiu YN, Huang AC, Chien YH, Hwu WL, Chiu MJ: Blood Beta-Amyloid and Tau in Down Syndrome: A Comparison with Alzheimer's Disease. Front Aging Neurosci 2016, 8:316.

47. Coppus AM, Schuur M, Vergeer J, Janssens AC, Oostra BA, Verbeek MM, van Duijn CM: Plasma beta amyloid and the risk of Alzheimer's disease in Down syndrome. Neurobiol Aging 2012, 33:1988-1994.

48. Strydom A, Heslegrave A, Startin CM, Mok KY, Hardy J, Groet J, Nizetic D, Zetterberg H: Neurofilament light as a blood biomarker for neurodegeneration in Down syndrome. Alzheimers Res Ther 2018, 10:39.

\section{Supplementary Information}

Additional File 1: Supplemental Table 1. Demographics of the PRECISION study cohort. Supplemental Table 2. Individual results of plasma Aß42, NT1 tau, and NfL in Down syndrome and controls.

Supplemental Table 3. Change-point regression for association of age with plasma biomarker levels.

Additional File 2: Supplemental Figure 1. Plasma $A \beta 42$ levels are stable when measured in the same subjects over a 5 day period. Supplemental Figure 2. Plasma NT1 tau levels are stable in the same subjects over a 5 day period. Supplemental Figure 3. Plasma NfL levels are stable in the same subjects over a 5 day period. Supplemental Figure 4. Prediction of biomarker changes by age using piece-wise linear regression.

\section{Figures}

A

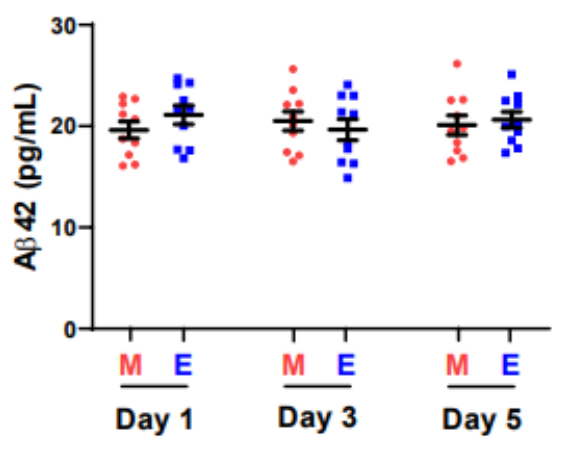

B

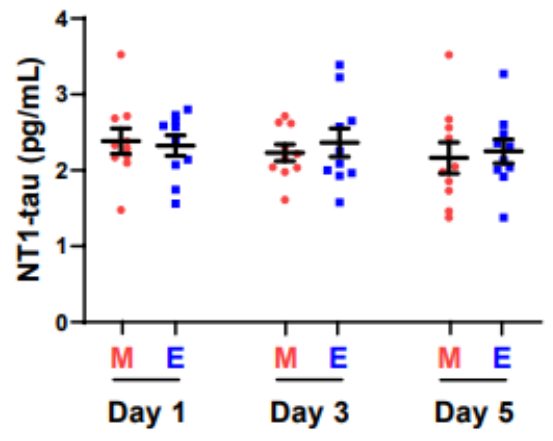

C

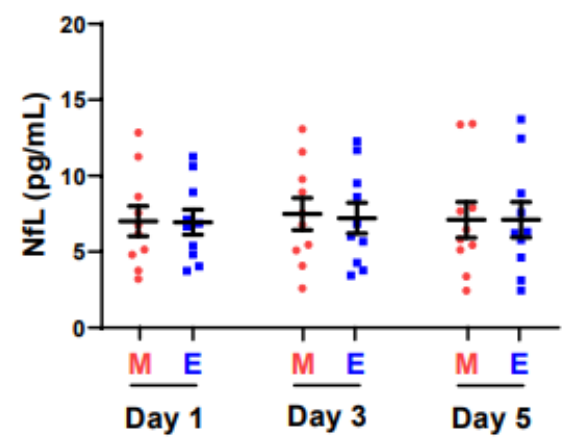

Figure 1 
Plasma A 42 , NT1 tau and NfL levels are constant over a 5 day period. Plasma was collected from 10 healthy volunteers on three occasions at 2 day intervals (day 1, day 3, day 5). On each sample day, blood was collected at 2 time points: the first between 7 - $10 \mathrm{am}(\mathrm{M})$, and the second between $4-7 \mathrm{pm}(\mathrm{E})$. Specimens were analyzed using ultra-sensitive Simoa-based assays for (A) A 42 , (B) NT1 tau and (C) NfL. Shown are measurements for each of the analytes on day 1 , day 3 , and day 5 . Each point represents a single measurement, and mean \pm SEM are indicated. Values measured in morning samples $(M)$ are in red circles, and evening specimens (E) are in blue squares. Mixed effect regression analysis revealed that plasma levels of (A) A 42 , (B) NT1 tau, and (C) NfL were stable and not significantly influenced by time of day (morning vs. evening) and did not change over a 5 day interval (Day 1 vs. Day 3 vs. Day 5).

A

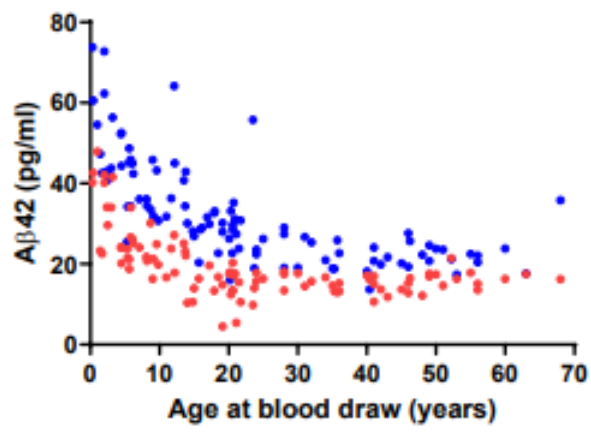

D

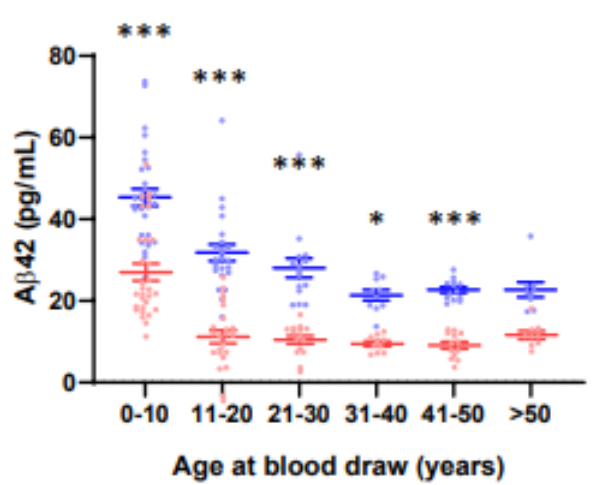

B

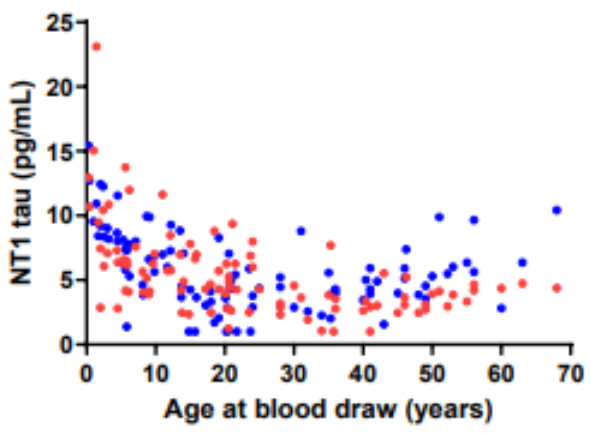

$\mathbf{E}$

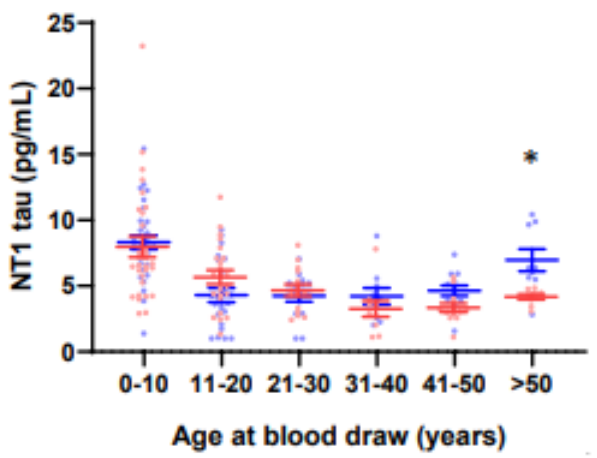

C

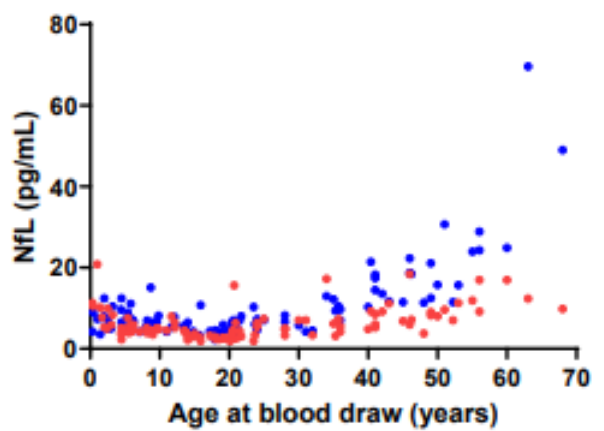

$\mathbf{F}$

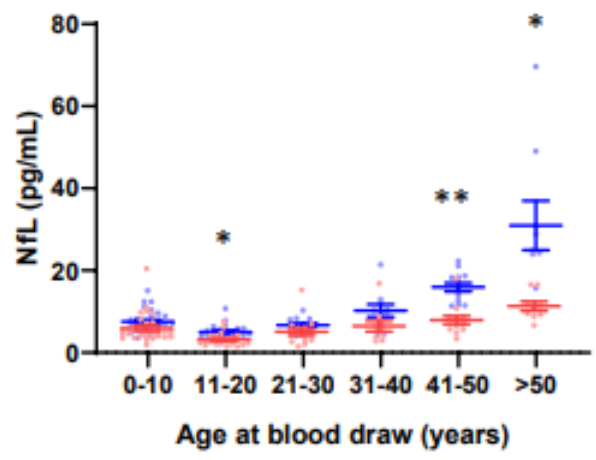

Figure 2

Plasma Aß42, NT1 tau, and NfL change differentially with age in Down syndrome versus control subjects. Plasma samples from 100 individuals with DS and 100 controls matched for age and sex were analyzed with ultra-sensitive assays for (A + D) A 442, (B + E) NT1 tau, and (C + F) NfL. (A - C) Individual A 42 , NT1 tau, and NfL values of subjects with DS (blue) and controls (red) are shown as a function of age. Each point represents one individual. (D - F) Data displayed in A - C are grouped in 10 year intervals. Each open circle represents a single individual. Mean and standard error of the mean are shown. $(A+D)$ At any given age, the absolute amount of $A \beta 42$ is higher in DS than in control subjects, but levels of $A \beta 42$ decrease with age, with a greater decrease in DS. $(B+E) N T 1$ tau levels tend to increase in older individuals with $\mathrm{DS}$ compared to controls. $(\mathrm{C}+\mathrm{F}) \mathrm{NfL}$ levels show a greater increase with age in 
individuals with DS than controls. Differences were assessed with paired t-test (normally distributed data) or Wilcoxon signed-rank test (non-normally distributed data). p-values were adjusted for multiple testing using Bonferroni correction. ${ }^{*} p<0.05 ;{ }^{* *} p<0.01 ;{ }^{* *} p<0.001$.

A

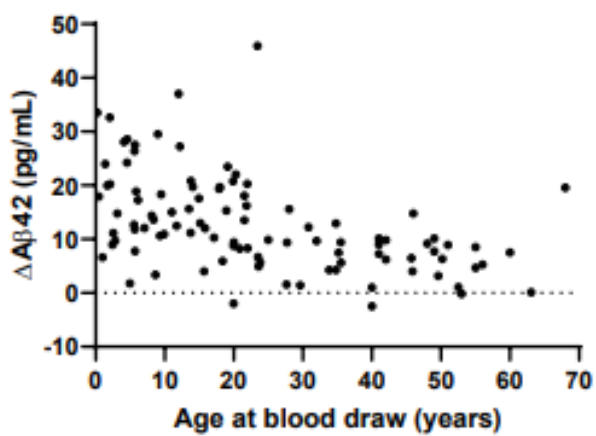

D

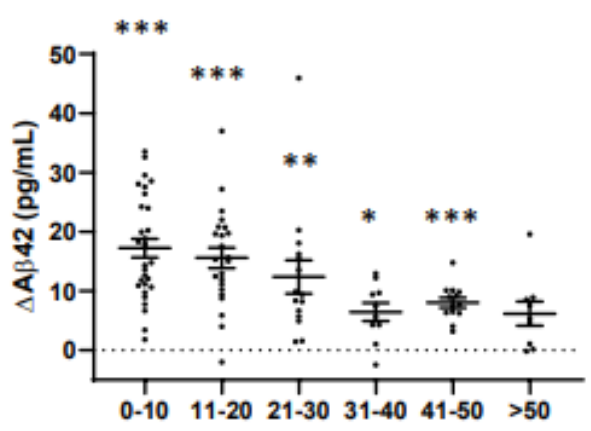

Age at blood draw (years)
B

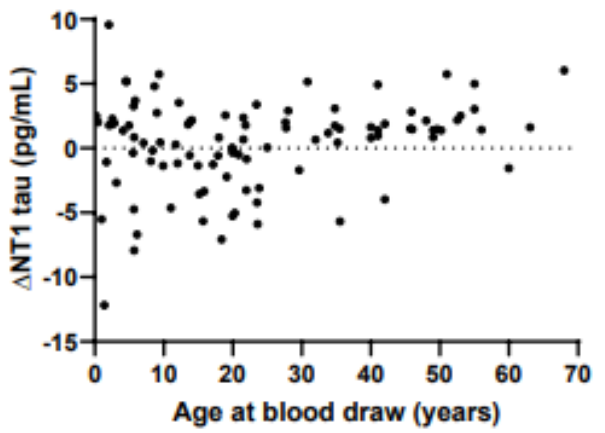

E

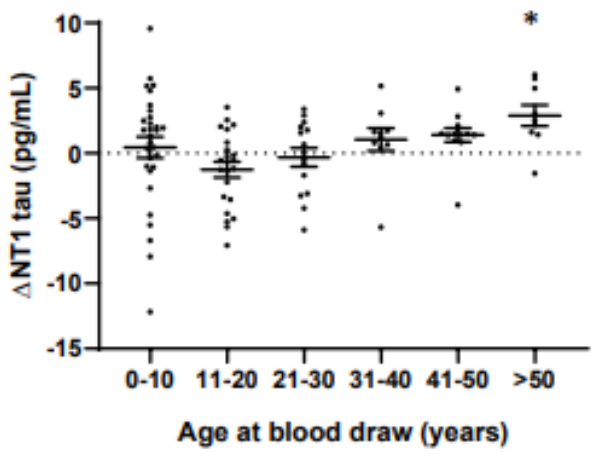

C

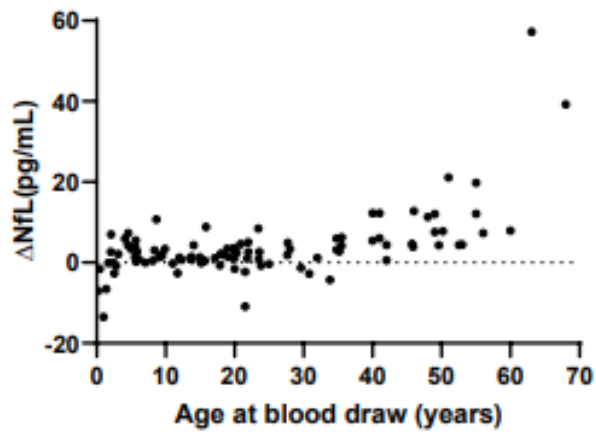

$\mathbf{F}$

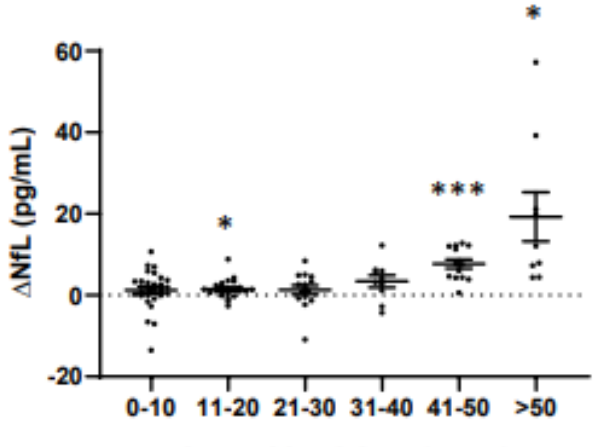

Age at blood draw (years)

Figure 3

Relative levels of plasma AB42 decrease in DS with age, whereas the relative levels of NT1 tau and NfL tend to increase. To compare relative changes of plasma biomarkers in DS compared to controls we calculated the difference between each sex- and age-matched pair ( $\triangle=D S-$ control). For (A) $\triangle A \beta 42$, (B) $\triangle N T 1$ tau, and (C) $\Delta N f L$, each point represents a single difference value. (D - F) Difference values are also grouped in 10-year intervals. Each open circle represents a single individual. Mean and standard error of the group-mean are shown. $(A+D) A \beta 42$ decreases with age in DS. $(B+E) N T 1$ tau is highly variable in the first 3 decades of life, but tends to increase in later life in DS. (C $+F) \Delta N f L$ is relatively constant in the first 30 years of life, but increases in DS with age. Differences were assessed with one sample t-test (normally distributed data) or one sample Wilcoxon signed-rank test (non-normally distributed data). $p$-values were adjusted for multiple testing using Bonferroni correction. ${ }^{*} p<0.05$; ${ }^{\star *} p<0.01 ; * \star * p<0.001$.

\section{Supplementary Files}


This is a list of supplementary files associated with this preprint. Click to download.

- SupplementalFigures14Jan27.pdf

- AdditionaldataforReviewJan27.docx

- SupplementalTables13Jan27.docx 